\title{
ACTITUDES HACIA LA COMPRA DE INTANGIBLES A TRAVÉS DE INTERNET EN ESTUDIANTES CIBERNAUTAS DE LA UNMSM
}

\author{
ATTITUDES TOWARD THE PURCHASE OVER THE INTERNET ON CYBERNAUT \\ STUDENTS OF UNMSM
}

\author{
Susan Pérez $A^{1}$ \\ Universidad Nacional Mayor de San Marcos, Lima, Perú \\ (RECiBido el 05/09/2011, ACEPTADO El 2/12/ 2011)
}

\begin{abstract}
Resumen
El desarrollo de la tecnología informática plantea la introducción de plataformas virtuales para realizar compras a través de Internet, las cuales generan beneficios tanto a las empresas, como a los consumidores; sin embargo, este tipo de compra no está difundida aún en el Perú. Es por ello que, considerando que las actitudes implican una evaluación de un objeto que orienta hacia la acción (La Rosa, 1983), se ha tomado a las actitudes hacia este medio de comercialización, como predictores del comportamiento de los internautas para efectuar o repetir una compra on-line. Así, con la finalidad de conocer el nivel de aproximación o rechazo (actitudes) hacia las compras on-line, que existe en una muestra de estudiantes universitarios cibernautas, se diseñó una escala de actitudes hacia la compra a través de Internet que consta de 16 ítemes, distribuidos en dos dimensiones: ventajas percibidas y evaluación del riesgo, teniendo en cuenta, el modelo explicativo de adopción de innovaciones de Schiffman y Kanuk (2000), según el cual estas dos dimensiones consideradas en la escala de actitudes, son valoradas por la persona para poder tomar una decisión que lo lleve a adoptar, postergar o rechazar una innovación, que en este caso es la compra virtual. La escala fue respondida por 107 personas y los datos revelaron mayor incidencia en niveles de actitud que expresan sentimientos de aproximación hacia la compra a través de Internet en cuanto a percepción de beneficios; sin embargo, sucede lo contrario en lo que respecta a la evaluación del riesgo.
\end{abstract}

Palabras clave: actitudes, compra a través de Internet, innovación, ventaja percibida, evaluación del riesgo.

\section{ABSTRACT}

The development of informatic technology introduces virtual platforms to purchase over the Internet, which generate benefits for both businesses and consumers, but this type of purchase is not widespread in Peru yet. That is why, taking in account that attitudes involves an assessment of an object and orients behaviour (La Rosa, 1983), we have taken the attitudes towards this form of marketing, as a predictor of Internet users to make or repeat a purchase

1 Estudiante del X ciclo de Psicología de la Universidad Nacional Mayor de San Marcos E-maill: susanperezal@gmail.com 
on-line. Thus, in order to know the level of approximation or rejection (attitudes) toward purchases on-line, which exists in a sample of college students Internet, we designed a range of attitudes towards the purchase through the Internet. It has 16 items, distributed in two dimensions: advantages perceived and risk assessment, taking into account the explanatory model of adoption of innovations developed for kanuka Schiffman (2000), whereby these two dimensions considered in the range of attitudes, are valued by person to make a decision to adopt, postpone or reject an innovation, which in this case is the virtual purchase. The questionnaire was answered by 107 people and the data revealed higher incidence levels of attitude that expresses feelings of approach toward the purchase over the Internet in terms of perceived benefits, but the opposite is for assessment of risk.

Key words: attitudes, purchase over the Internet, innovation, perceived benefit, assessment of risk.

\section{INTRODUCCIÓN}

La globalización, en tanto proceso económico, político, social y cultural ha insertado diversas modificaciones en el mundo en el que nos desenvolvemos, algunas de ellas son las nuevas técnicas de administración, gestión y comercialización. Para ello, un impulsor e incluso decisor de vital importancia, ha sido el avance de la tecnología, principalmente la informática, gracias al exitoso desarrollo de la Internet: una herramienta que favorece la educación, la comunicación, el comercio, la expansión de mercados, entre otros campos.

La rápida difusión de estas nuevas tecnologías se traduce en 2,095,006,005 usuarios de internet en el mundo, lo que equivale a un $30,2 \%$ del total de la población, de la cual un 10,3\% corresponde a América Latina y el Caribe (Internet World Stats, 2011). En nuestro país, el panorama es favorable, ya que el Perú, junto a Brasil y Argentina, cuenta con una de las tasas más altas de uso de Internet en la región. Particularmente, contamos con 4.2 millones de usuarios, donde predominan cibernautas entre 25 y 34 años de edad, alcanzando un $32 \%$ del total, seguido de un $24 \%$ correspondiente a jóvenes entre los 14 y los 24 años, siendo este último grupo el que pasa mayor tiempo en Internet. Al respecto, contamos con un uso promedio de 26.9 horas mensuales por usuario, lo que equivale a tres horas más que el promedio mundial (Fosk, A., 2011).

Figura 1. Usuarios de Internet en América Latina y total de horas online mensuales por visitante en América Latina.
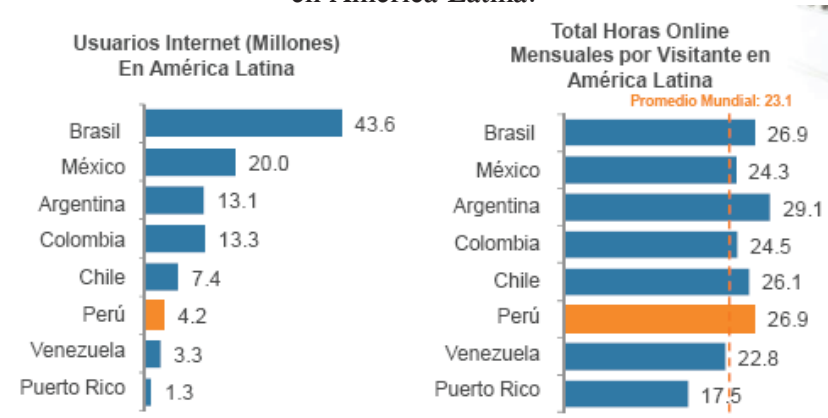

Fuente: Estado de Internet con un Enfoque en el Perú. Comscore. 2011. 
Internet permite a las personas reunirse en un espacio virtual, asociarse en comunidades, comunicarse en tiempo real sin importar las barreras planteadas por la distancia y usar otras herramientas digitales diversas. Entonces, teniendo esta amplia gama de opciones, nace un nuevo espacio de desarrollo para las empresas, favoreciendo así el comercio, el cual ha adoptado las herramientas que le brinda la era informática.

El comercio ha existido desde tiempos remotos, incluso antes del desarrollo de las grandes civilizaciones, y ha sido útil para sobrevivir; sin embargo, ha ido evolucionando desde el trueque hasta la incursión de la moneda acuñada, para pasar luego a las tarjetas de crédito y el dinero electrónico (A. Villar, 2004).

La adopción de estas nuevas herramientas ofrecidas por las tecnologías informáticas se dio de manera gradual, por el comercio. Se inició con una etapa denominada Web estática, donde las empresas generaban una página web en la que daban a conocer los servicios o productos que ofrecían, reproducían sus catálogos, indicaban la ubicación de sus locales, sus números telefónicos de contacto y algunas promociones. No obstante, aún había ciertas deficiencias, pues era necesario ir hasta la tienda para hacer efectiva la compra, lo que acarreaba algunas inconveniencias al usuario, como movilizarse hasta el local, perdiendo tiempo; además, hacer colas, tener que ir a las tiendas en un horario de atención restringido, y otras adicionales. Por ello, se explotó aún más el desarrollo de la tecnología web, y se pasó a una siguiente etapa: la compra-venta on-line, donde los clientes han empezado a tener la oportunidad de comprar tangibles e intangibles a través de internet, haciendo uso de tarjetas de crédito o de dinero electrónico (A. Villar, 2004).

Actualmente, para usar una tarjeta de crédito por Internet, únicamente debemos digitar el número de la misma y una clave secreta, o un número impreso en la misma tarjeta. Existen otros sistemas como las claves temporales, generadas cada cierto tiempo por un dispositivo electrónico entregado por los bancos a sus clientes. También tenemos el efectivo digital, conocido como e-money o dinero electrónico.

Una de las empresas más conocidas que ofrecen servicios de esta naturaleza, es la estadounidense PayPal, que permite tener una cuenta en Internet, la cual es cargada con dinero de diferentes fuentes, ya sea una tarjeta de débito, de crédito o una cuenta bancaria. Este dinero es almacenado en una sola cuenta virtual y enviado, previa autorización del titular, a diferentes destinatarios: personas o tiendas que operan a través de Internet, cada vez que el usuario desee hacer una transferencia o compra, sin necesidad de que comparta información financiera en cada una de sus operaciones virtuales (Tomado de la página oficial de PayPal: www.paypal.com. es ). Del mismo modo, la empresa originaria de Hong Kong: Octopus, opera con dinero electrónico, pero con un sistema diferente. Los usuarios depositan dinero a la tarjeta Octopus Limited, y esta empresa se encarga de depositarlo nuevamente en bancos, permitiendo que esta tarjeta tenga un sistema de funcionamiento similar a las de débito (Tomado de la página oficial de Octopus: www.octopus.com.hk ).

Además de las facilidades de pago virtuales, el comercio electrónico ofrece a los usuarios una amplia gama de beneficios, pues el consumidor tiene la posibilidad de 
comunicarse con un agente de la empresa, quien lo guía en su compra, resolviendo sus dudas y brindándole asesoría a través de sesiones de chat iniciadas en la página web del ofertante. Además, le brinda la posibilidad de compartir en foros, sus opiniones y experiencias con otros consumidores, respecto del producto que está pensando adquirir o que ya ha adquirido. También, se ha traslado al Internet, esquemas tradicionales como la subasta o el remate, donde un vendedor oferta un producto en la red, y varios compradores potenciales compiten entres sí, declarando el precio que está dispuesto a pagar, teniendo además, la posibilidad de conocer los precios ofrecidos por otros posibles compradores, hasta que en determinado momento el vendedor ejecuta la venta a un precio que le resulta favorable. Este mecanismo de compra lo encontramos, por ejemplo, en www.ebay.com y www. amazon.com. Adicionalmente, Internet, en tanto medio de comunicación, permite al comprador asociarse con otras personas interesadas en adquirir un mismo producto, y solicitarlo en un volumen mayor, de manera que obtienen un descuento. Así, el precio final de cada producto, resulta siendo menor al que hubieran resultado si cada uno realizaba una compra por separado. Por último, el consumidor tiene la opción de obtener comprobantes de pago electrónicos y archivarlos de manera ordenada, comparar precios en diferentes tiendas virtuales a través de aplicaciones especiales para ello, comprar artículos que no se encuentran disponibles en su ciudad de residencia o realizar compras las 24 horas del día, los 365 días del año.

Para las empresas, Internet es un recurso útil para reforzar o complementar los canales de venta tradicionales. Además, vender en línea implica la apertura al mercado mundial, un mercado de expansión ilimitada; brinda la posibilidad de disminuir costos al eliminar intermediareios, gracias al trato directo con el cliente y la necesidad de un número reducido de empleados para el procesamiento de los pedidos. Por otro lado, brinda la oportunidad de incrementar los canales de comunicación con sus clientes y mejorar su prestigio y reconocimiento al contar con una tienda virtual.

Vemos entonces, que el comercio electrónico o e-commerce, en sus diferentes modalidades, ha venido funcionando como una nueva forma de dirigirse a los clientes y potenciales clientes, como un nuevo canal de distribución y como un revolucionario de los hábitos de consumo.

Ahora bien, el comercio electrónico ofrece una serie de beneficios, antes expuestos; sin embargo, también trae algunos inconvenientes tales como la contratación de un especialista que cree la página web y una inversión constante en la actualización y mantenimiento de la página web de la empresa. Pero los principales enemigos del e-commerce son los hackers, que valiéndose de desarrollada tecnología, pueden llegar a tener acceso a servidores, clonar páginas web, capturar las claves de los usuarios, entre otros.

Pese a ello, cada vez más y más personas alrededor del mundo compran a través de Internet, pues gracias a sus beneficios ha tenido una muy buena acogida; por ejemplo, en los Estados Unidos sólo hacia medio año del 2003, las compras a través de Internet ascendieron a 47500 millones de dólares (Lanzillota, 2007). No 
obstante, debemos mencionar que el panorama en América Latina, y en especial en el Perú, es distinto pues no alcanzamos altos porcentajes de usuarios de Internet que ejecuten compras a través de este medio. Ello se refleja en los 276 millones de dólares que alcanzamos en ventas por Internet en el 2009 (Fosk, A., 2011).

En nuestro país, actualmente ya contamos con diferentes herramientas que favorecen la compra a través de Internet. Los bancos han implementaron transacciones bancarias a través de sus páginas web, lo mismo sucedió con la SUNAT y su sistema de pagos en línea, y algunos cines que permiten comprar tickets en sus portales web para evitar las colas en las boleterías.

Este desarrollo también se puede apreciar en el interés que tienen los peruanos por visitar páginas web que permiten entablar relaciones comerciales en entornos virtuales. De hecho, las cifras que nos presenta A. Fosk (2011) nos indican que efectivamente un grupo de peruanos busca comprar a través de Internet, compara precios y conoce dónde encontrar proveedores en línea. Tenemos que en el país, un 57\% de usuarios de Internet visitan webs de retails, siendo Mercado Libre y Amazon, las páginas más visitadas. Sin embargo, esta cifra se encuentra por debajo del promedio mundial (65\%). Adicionalmente, el Perú invierte un promedio de 25.5 minutos en visitas a páginas de retails al mes, frente a un 35.4 en América Latina, y a un 57.1 a nivel mundial (Fosk, A., 2011), tal como lo podemos apreciar en el gráfico 2 ..

Figura 2. Principales sitios de retail en Perú.

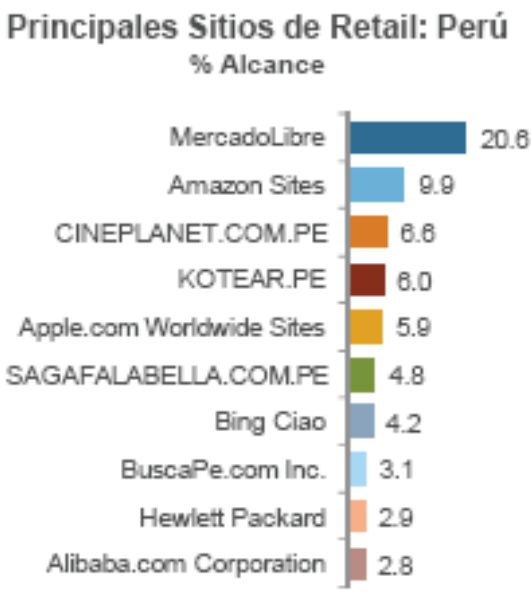

Fuente: Estado de Internet con un Enfoque en el Perú. Comscore. 2011

Se estima que el comercio electrónico continuará creciendo durante este año en un $40 \%$. Para ello, cada vez más empresas, de diferentes rubros, deberán implementar plataformas virtuales de comercialización, pues actualmente del total de empresas que ya lo han hecho, un $22 \%$ corresponde a flores y regalos, un $13 \%$ a negocios relacionados al turismo, y un $8 \%$ pertenece a la venta de electrodomésticos (Fosk, A., 2011). 
Una herramienta útil para ello son las redes sociales, que se han convertido en un boom entre los jóvenes, lo que abre a las empresas una forma de llegar directamente hacia ellos a través de un medio apreciado y común entre la gente joven, quienes son los principales consumidores de Internet. En nuestro país, un $92 \%$ de los usuarios de Internet lo usa principalmente para tener acceso a redes sociales, lo que se traduce en 9 peruanos de cada 10, que actualmente usan redes sociales (Fosk, A., 2011).

Ante la aparición de nuevas plataformas de compra-venta en el mercado, las pautas de comportamiento de los consumidores pueden llegar a ser muy distintas; así, algunos asumen con gran facilidad las innovaciones tecnológicas, como lo es la compra on-line en este caso, mientras que otros no. Aquí, la ciencia llamada a dar explicación es la Psicología del Consumidor, una rama que nos brinda una mirada amplia y sistemática de la conducta del hombre en su desenvolvimiento en el mercado, resolviendo preguntas como: por qué muchos cibernautas no compran on-line, aún teniendo la posibilidad de hacerlo, o qué aspectos habrán de ser modificados para impulsar y promover la compra a través de Internet.

En Psicología del consumidor, las actitudes asumen un valor estratégico, en tanto que poseen un elemento motivacional que impulsa a la acción. Así lo señala Allport (Arellano, R., 2002), al definir las actitudes como "un estado mental y neurológico con capacidad de reacción, organizado por la experiencia, que ejerce una influencia directriz o dinámica sobre la respuesta del individuo frente a todos los objetos y situaciones con las cuales está en relación". En el caso de la Psicología del Consumidor, se ve que las actitudes impulsan a las personas hacia la adquisición de un producto o la adopción de un servicio (Arellano, R., 2002).

Vemos pues, que las actitudes van a determinar en gran manera la conducta de las personas (Baron A. et al, 1998); por ello, consideramos de gran importancia la realización de un estudio que nos provea de datos acerca de la dirección e intensidad de las actitudes hacia la compra a través de Internet, como puno de partida para ejercen una influencia notable sobre los bajos índices de adopción de compra a través de Internet que existen en nuestro país, ya que un consumidor, al poseer actitudes de rechazo hacia las compras on-line, inhibirá acciones que lo lleven a adquirir algún producto a través de medios informáticos, y sucederá lo contrario si posee actitudes favorables hacia ello.

Las actitudes son adquiridas en el proceso de socialización al interactuar en un contexto, que condiciona su naturaleza, son relativamente permanentes, se relacionan directamente con los objetos y poseen una cualidad direccional (positiva o negativa), que forma un patrón evaluativo al formar categorías complejas, las cuales se ponen en evidencia ante las distintas situaciones que vivimos.

Las actitudes también pueden formarse por comparación social, mecanismo que se basa en el concepto de comparación social. Baron et al (1998) citan a Festinger para definir este concepto como la tendencia a compararnos a nosotros mismos con los otros para determinar si nuestra visión de la realidad es correcta o no. De este modo, si al comparar nuestras actitudes hacia determinado estímulo, con 
las de los otros, vemos que coinciden, consideraremos que tenemos una actitud adecuada, o adoptaremos aquellas que priman en el grupo.

Las actitudes poseen un componente cognitivo, que hace referencia a las apreciaciones y opiniones que el objeto de actitud despierta en nosotros. Al respecto, Baron A. ,R. \& Byrne, D. (1998) afirma, citando a Wyer y Srull, que las actitudes funcionan como esquemas, o marcos cognitivos, los cuales poseen y organizan la información acerca de conceptos específicos, situaciones y acontecimientos. Así, las actitudes van a influir sobre cómo pensamos acerca de todo aquello con lo que nos topamos en nuestra interacción con la realidad, y en el modo cómo procesamos la información que tenemos de ello. Pero, para que todo aquello que implica el componente cognitivo, sea formado, no es necesario que el sujeto tenga un conocimiento amplio acerca del objeto hacia el que se dirige la actitud; además, éste puede ser real o erróneo, o puede basarse en un suceso impactante que ha vivenciado el sujeto, y ha despertado una opinión en él (La Rosa, 1983).

Las actitudes también poseen un componente afectivo, que está en relación a su valor emocional, el cual hace que el individuo experimente un determinado grado afectivo hacia el objeto de actitud, ya sea negativo o positivo, en base a las características conocidas del mismo. Al respecto, pueden presentarse conflictos de fuerzas afectivas contradictorias con respecto a dos características del mismo objeto de actitud. Frente a ello, la resolución de este conflicto se orientará a la característica que posee la mayor fuerza (Arellano, 2002). Este componente va a influenciar fuertemente sobre los otros dos componentes de las actitudes.

Los elementos que aportan los componentes cognitivo y afectivo han de exteriorizarse de alguna manera en la interacción con el contexto que ejerce el individuo, mediante la predisposición o intención a la acción como resultado de la valoración que se ha hecho del objeto de actitud.

Finalmente, las actitudes poseen un componente conductual, que le permite al sujeto exteriorizar el contenido de sus actitudes, cuando se encuentra en relación con su medio. Como bien lo expresa H.Triadis (citado por Arellano, R., 2002), la actitud es "una idea cargada de emoción que predispone a un tipo de acción frente a un tipo de situación específica".

En tal sentido, es posible predecir la conducta del individuo en contextos determinados, en base al conocimiento de sus actitudes. Así por ejemplo, las personas van a preferir dedicarse a una determinada actividad, integrarse a ciertos grupos o desenvolverse en espacios que se adecúen a sus actitudes.

Sin embargo; en muchas ocasiones, existe una amplia brecha entre las actitudes y la ejecución de acciones que obedezcan a las mismas. Por ello, la pregunta a resolver al estudiar las actitudes, ya no es si éstas influyen sobre el comportamiento, sino ¿Cómo y cuándo ejercen esta influencia? (Baron A., R. \& Byrne, D., 1998), para conocer de qué factores va a depender que exista correspondencia entre las actitudes y la conducta. 
Uno de estos factores son las restricciones situacionales. Sucede que una persona puede poseer una actitud específica con relación a algún estímulo; sin embargo, la situación en la que se encuentra no le permite expresarla, ya sea porque iría en contra de las normas, las convenciones sociales, etc.

Otro factor es la presión temporal. Baron A.,R. \& Byrne, D.(1998) citan a Jamieson y Zanna para indicar que las actitudes, en tanto que son un marco de referencia, ejercen una influencia bastante fuerte cuando una persona ha de tomar decisiones de manera rápida, llevándola a actuar de acuerdo a determinada actitud o componente de la actitud que se presenta más rápidamente a la conciencia. No sucede lo mismo, cuando se cuenta con mayor tiempo para hacer un análisis más exhaustivo de la información que se posee.

También influye el origen de las actitudes. Aspectos relativos al origen de las actitudes intervienen en la relación actitud-conducta. Así, las actitudes que han sido formadas gracias a una experiencia directa, se mantienen más presente en el individuo, y por tanto es más probable que éstas influyan sobre su conducta.

Finalmente un factor decisivo es la intensidad de las actitudes. Baron A., R. \& Byrne, D. (1998) citan a Petkova, Ajzen \& Drivers, quienes sostienen que las actitudes más fuertes son las que tienen mayor impacto sobre el comportamiento. La intensidad de las actitudes implica diversos factores que van a ser los que determinarán la intensidad o extremo al que llegue una actitud. Así, una actitud intensa, será más difícil de modificar, perdurará más en el tiempo y formará una parte importante de la cognición social.

Al respecto, existen algunos elementos que influyen sobre la intensidad de una actitud, los cuales repercutirán luego, sobre la actuación del sujeto. Estos son: la importancia, el conocimiento y la accesibilidad. El primero hace referencia a cómo afecta personalmente la actitud al sujeto, o cuánto se preocupa ésta por una actitud, o cómo afecta sobre el interés del sujeto. Así, la importancia de una actitud afectará en la toma de decisiones del sujeto, en el modo como procese información, y sobre las acciones que realice. El segundo se refiere a que la medida en la que el sujeto va a actuar correspondiendo a sus actitudes depende de cuánto sepa con respecto al objeto de actitud. Por último, el tercer elemento se refiere a la facilidad con la que una actitud puede ser llevada a la mente, en diversas situaciones.

Por otro lado, Baron A., R. \& Byrne, D. (2005) nos recuerdan la Teoría de acción razonada propuesta por Ajzen y Fishbein, como un importante aporte para explicar cómo las actitudes influyen sobre el comportamiento. Según esta teoría, las decisiones que tomamos con respecto a emitir una conducta específica, se da como resultado de todo un proceso racional, que sigue un orden lógico y se orienta hacia una meta. Este proceso permite al sujeto, evaluar las opciones de la conducta, las consecuencias de llevarlas a cabo, para finalmente tomar la decisión de realizarlas o no.

Para lograr un cambio de actitudes, se puede actuar sobre uno, o todos, sus componentes (Arellano C., R. ,2002). A continuación se indica cómo se da el 
cambio según el componente sobre el que actúa.

A partir de un elemento cognitivo, el cambio se da al brindar información al sujeto, con respecto al objeto de actitud. Esta información puede ser nueva, complementaria a la que ya manejaba, e incluso, contradictoria a la misma.

A partir de un elemento afectivo, se apela al mecanismo de emisión de mensajes con mayor sustento e impacto emocional que el que caracteriza a la actitud actual, promoviendo así una confrontación entre dos actitudes de tal forma que sobresale la que se busca proponer. El cambio se da sin la necesidad de que el sujeto reciba información nueva acerca del objeto de actitud (Arellano C., R. ,2002).

Para realizar un cambio de actitudes a partir de un elemento conductual, el individuo ha de estar inmerso en una situación que lo obligue o impulse a la acción. Podríamos indicar un ejemplo que englobe a los tres componentes de la actitud, haciendo mención de una campaña que difunda información acerca de los mecanismos de contagio del SIDA, y las conductas que no son vía de contagio (elemento cognitivo). Luego, se publicita esta campaña por los diversos medios de comunicación, siendo muchos líderes de opinión quienes la difundan y sean imágenes de dicha iniciativa (elemento afectivo). Finalmente se organiza un evento cultural donde personas portadoras comparten espacio físico en un establecimiento (elemento conductual).

Otra forma de analizar la adopción o rechazo de la compra a través de Internet por parte de los consumidores, es recurriendo al modelo explicativo de adopción de innovaciones de Schiffman y Kanuk (2000), quienes presentan estudios de cómo afectan sobre la adopción de una innovación, factores evaluativos como la complejidad percibida, las ventajas percibidas, los atributos de la innovación, la compatibilidad con el estilo de vida y el riesgo percibido. Siguiendo esta línea de investigación, podríamos considerar a la compra on-line como una innovación que el consumidor puede rechazar, postergar o adoptar, en base a cómo la evalúa, lo que determinará su actitud hacia la misma al generar, para finalmente condicionar su conducta. Tenemos que si evalúa que los beneficios superan a los costes que implica la compra on-line, tales como el tener que familiarizarse con plataformas electrónicas o el riesgo de hacer una transacción económica on-line, sus actitudes serán favorables y tendrá mayor probabilidad de emitir una conducta de compra.

En base a lo expuesto anteriormente, planteamos el estudio de las actitudes hacia la compra a través de internet como un elemento clave para el desarrollo del mercado virtual y la identificación de elementos sobre los cuales trabajar para extender el alcance de este medio de comercialización.

Se va a evaluar las actitudes hacia la compra a través de Internet teniendo en cuenta, el modelo explicativo de adopción de innovaciones de Schiffman y Kanuk (2000), que nos provee elementos de análisis del objeto de actitud que vamos a evaluar. Estos elementos de análisis son representados por los dos indicadores del instrumento de evaluación aplicado para este estudio: las ventajas percibidas y la evaluación del riesgo. Según este modelo, las personas valoran estos elementos 
antes de tomar, postergas o rechazar la adopción de una innovación, que en este caso es la compra virtual. Estos elementos de análisis nos proveen de conocimiento de factores que determinan la formación de actitudes hacia la compra a través de Internet, lo cual es objeto de estudio de la presente investigación.

Se ha elegido los dos indicadores mencionados porque consideramos que son dos aspectos que caracterizan la compra on-line y que la diferencian de la compra off-line o tradicional. En ese sentido, vemos que en el proceso decisional de la compra, el consumidor hará una evaluación de los beneficios y los riesgos que asume cuando realiza una transacción comercial virtual.

Así, los objetivos de este trabajo para conocer las actitudes hacia la compra de intangibles a través de Internet se centran en el conocimiento de la dirección y el nivel de las actitudes de los estudiantes cibernautas de la UNMSM. Entonces, nos planteamos la pregunta: ¿Cuál es la dirección y nivel de las actitudes hacia la compra de intangibles a través de Internet en estudiantes cibernautas de la UNMSM?

\section{METODOLOGÍA}

Estudio descriptivo transversal realizado entre abril y noviembre del 2010; mediante un muestreo por conveniencia, en el que se aplicó la Escala de actitudes hacia la compra a través de Internet (instrumento elaborado por el autor para fines de la investigación), previo consentimiento informado. En una población de 27986 alumnos de la Universidad Nacional Mayor de San Marcos, se seleccionó a 107, cuya distribución se puede apreciar en las tablas 1, 2 y 3.

Tabla 1. Distribución de la muestra según sexo y edad de los sujetos

\begin{tabular}{cccc}
\hline & Frecuencia & Porcentaje & $\begin{array}{c}\text { Media de la } \\
\text { edad }\end{array}$ \\
\hline Varones & 50 & $46.7 \%$ & 21.8 \\
Mujeres & 57 & $53.3 \%$ & 21.4 \\
Total & 107 & $100 \%$ & 21.6 \\
\hline
\end{tabular}

Tabla 2. Distribución de la muestra según año de estudios de los sujetos

\begin{tabular}{ccc}
\hline Año de estudios & Frecuencia & Porcentaje \\
\hline Entre $1^{\circ}$ y $3^{\circ}$ año & 33 & $30.8 \%$ \\
Entre $4^{\circ}$ y $5^{\circ}$ año & 74 & $69.2 \%$ \\
Total & 107 & $100 \%$ \\
\hline
\end{tabular}

Tabla 3. Distribución de la muestra según la especialidad académica a la que pertenecen los sujetos

\begin{tabular}{ccc}
\hline Especialidad & Frecuencia & Porcentaje \\
\hline Psicología & 73 & $68.22 \%$ \\
Ciencias administrativas & 15 & $14.02 \%$ \\
Ingeniería Mecánica & 19 & $17.76 \%$ \\
Total & 107 & $100 \%$ \\
\hline
\end{tabular}


Se elaboró una escala tipo Likert de actitudes hacia la compra a través de Internet, que consta de 16 items; 7 de ellos miden las ventajas percibidas del uso de medios virtuales como canal de compra, y los otros 9 miden la evaluación del riesgo de las compras a través de Internet. En la tabla 4 se puede observar la distribución de los items para cada indicador.

Tabla 4. Distribución de los items para cada indicador.

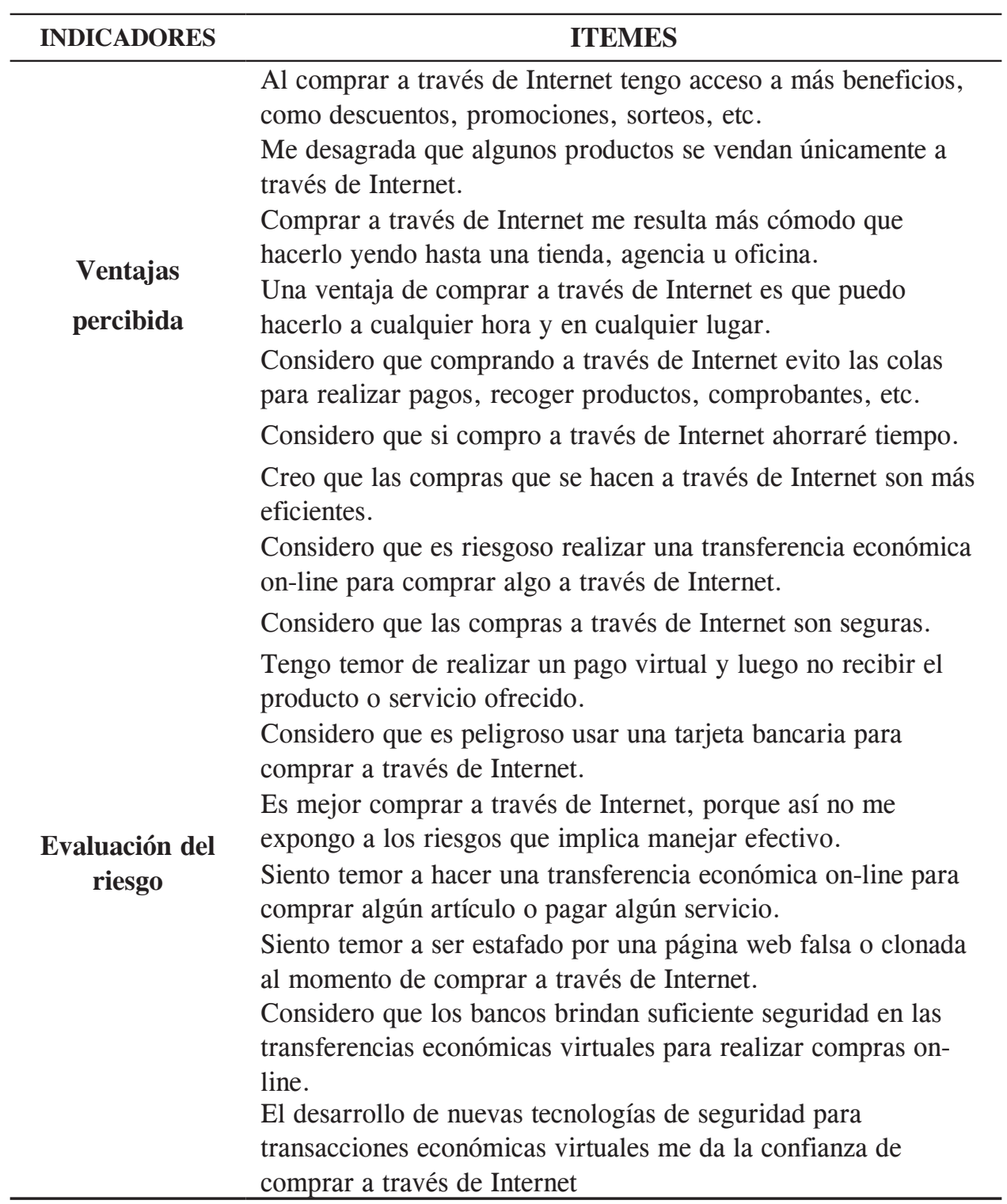


La escala otorgó una puntuación por ítem de 1 a 6 que variaba desde definitivamente en desacuerdo hasta definitivamente de acuerdo. Se determinó la confiabilidad del instrumento a través del coeficiente de consistencia interna alfa de Cronbach, con un valor de 0,91 . Los puntajes directos fueron transformados a puntuaciones $t \mathrm{y}$ clasificados como muestra la tabla 5. Se diferencia los niveles de aproximación o rechazo hacia la compra a través de Internet en categorías, según rangos de puntuaciones.

Tabla 5. Baremo de la Escala de actitudes hacia la compra a través de Internet

\begin{tabular}{cc}
\hline CATEGORÍA & RANGO DE PUNTUACIONES \\
\hline Muy desfavorables & $20-29$ \\
Desfavorables & $30-39$ \\
Tendencia al rechazo & $40-49$ \\
Tendencia a la aceptación & $50-59$ \\
Favorable & $60-69$ \\
Muy favorable & $70-80$ \\
\hline
\end{tabular}

\section{RESULTADOS}

En la figura 3 se muestra los valores medios alcanzados por cada ítem.

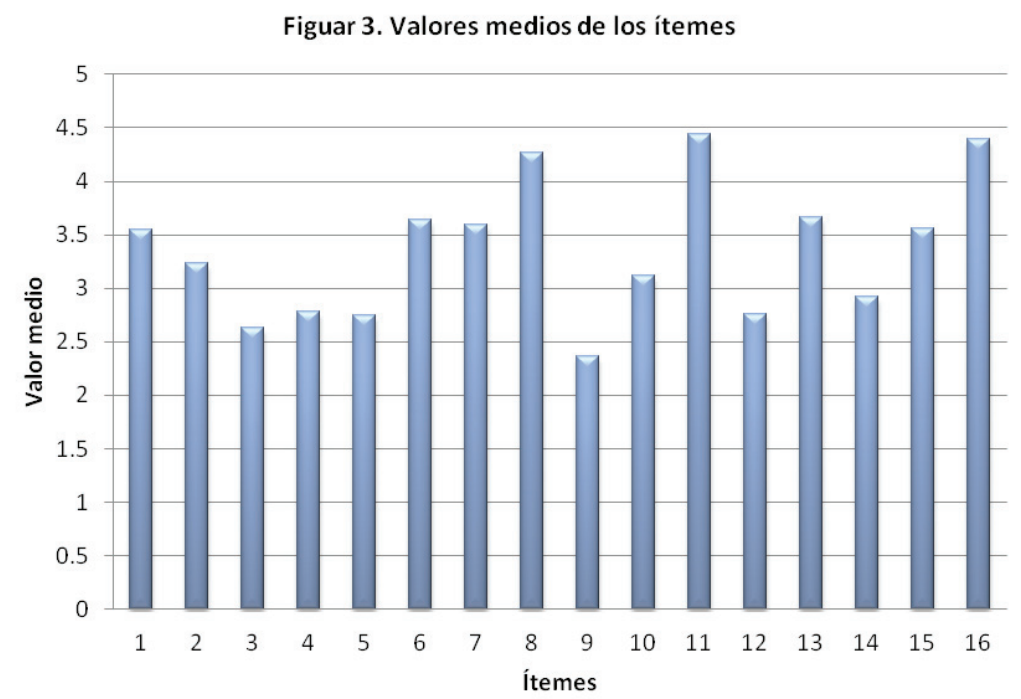

Las actitudes hacia la compra a través de Internet que alcanzaron mayor frecuencia fueron: comprando a través de Internet evito las colas para realizar pagos, recoger productos, comprobantes, etc. (71.7\%), Una ventaja de comprar a través de Internet es que puedo hacerlo a cualquier hora y en cualquier lugar (73.7\%) y considero que si compro a través de Internet ahorraré tiempo (73.3\%). 
Por el contrario, las actitudes que fueron menos frecuentes fueron: comprar a través de internet me resulta más cómodo que hacerlo yendo a hasta una tienda, agencia u oficina (40\%), tengo temor de realizar un pago virtual y luego no recibir el producto o servicio ofrecido $(43.3 \%)$, siento temor de hacer una transferencia económica on-line para comprar algún artículo o pagar algún servicio $(45 \%)$ y Me desagrada que algunos productos se vendan únicamente a través de Internet $(46.7 \%)$.

Según se observa en el gráfico 2, el $49 \%$ de los encuestados presentó actitudes con tendencia al rechazo de la compra a través de Internet, con una media de 45.4; mientras que el $27 \%$, mostró una tendencia a la aceptación, con una media de 54.3. Tanto las actitudes favorables como las desfavorables alcanzaron un $9 \%$ de la muestra, con una media de 63.3 y 36.1 respectivamente, un 5\% presentó actitudes muy favorables, con una media de 77.5 y solo un $1 \%$ mostró una actitud muy desfavorable, con una media de 26.8.

Figura 4. Distribución de la muestra según sus actitudes hacia la compra a través de Internet

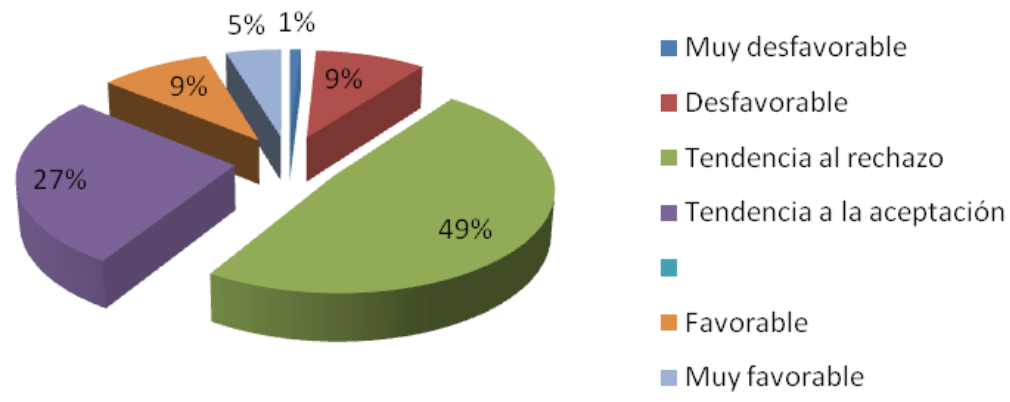

El análisis de correlación de Spearman muestra que ni la edad ni el sexo se correlacionan con las actitudes hacia la compra a través de Internet, como se puede observar en el gráfico 3.

Figura 5. Correlación entre edad y actitudes hacia la compra a través de Internet

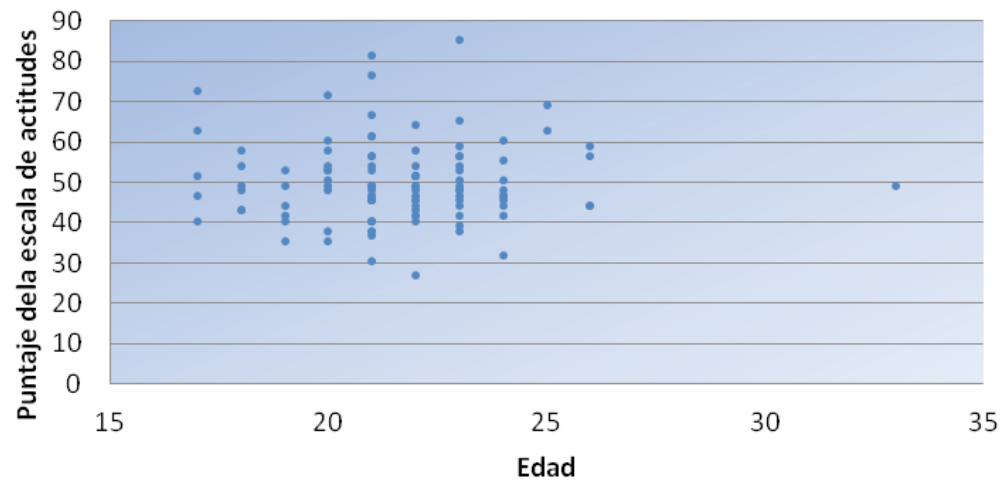




\section{DISCUSIÓN}

Las actitudes hacia la compra a través de Internet en los estudiantes de la UNMSM mostraron altas frecuencias y niveles de actitud que expresan sentimientos de aproximación, al momento de calificar las compras on-line en cuanto a las ventajas que ofrece este canal, principalmente en lo que respecta al ahorro de tiempo, la evitación de colas y la comodidad de realizar compras sin tener que ajustarse a los horarios de las tiendas o tener que movilizarse hacia ellas. Sin embargo, alcanzaron frecuencias bajas y niveles de actitud que expresan sentimientos de rechazo, al momento de evaluar los riegos que implica este tipo de transacción económica, primordialmente en lo concerniente a la ejecución de pagos en línea, dado que ello implica revelar cierta información acerca de tarjetas bancarias, lo que implica un riesgo ante páginas web clonadas, hackers, fishing, etc. De la misma manera evalúan el riesgo de fraude por parte de la empresa vendedora.

Las actitudes favorables encontradas en la muestra recaen sobre la mayoría de los ítemes provenientes del indicador "ventaja percibida" y las actitudes desfavorables recaen sobre la mayoría de los ítemes provenientes del indicador "evaluación del riesgo"; así, se observa, tal como lo plantearan Schiffman y Kanuk (2002), que para asumir una innovación, que en este caso es la compra a través de internet, un modo de compra ampliamente diferente del modo off-line tradicional, la persona hace evaluaciones tanto de los beneficios y los riesgos que implica. Así, vemos que no resulta difícil para la muestra reconocer los beneficios de la compra on-line, lo que deriva en actitudes positivas hacia ella. Sin embargo, es el riesgo percibido, lo que plantea una brecha entre los internautas y la adopción de la conducta de comprar a través de Internet.

Entonces, atendiendo a los beneficios que representa para una empresa su presencia en Internet y el desarrollo de plataformas virtuales de comercialización, creemos que para desarrollarlas es necesario trabajando sobre el componente cognitivo de las actitudes, ya que muchas de las valoraciones negativas que se hace sobre la compra on-line, se derivan del desconocimiento de cómo llevar a cabo una compra segura. Esto, por supuesto, además de desarrollar tecnologías que afirmen la seguridad de los sitios web y así conseguir un mayor desarrollo de esta herramienta en nuestro país. Es decir trabajar sobre la seguridad de la compra, dado que la gente ya percibe los beneficios del e-commerce. 


\section{REFERENCIAS BIBLIOGRÁFICAS}

Arellano C., R. (2002) Comportamiento del consumidor. Enfoque América Latina. México, D.F.: Mc Graw-Hill/Interamericana Editores, S.A. de C.V.

Bandura, A. \& Walters, R. (1990) Aprendizaje social y desarrollo de la personalidad. Madrid: Alianza Universidad.

Baron A., R. \& Byrne, D. (1998) Psicología Social. Madrid: Prentice Hall Iberia.

Baron A., R. \& Byrne, D. (2005) Psicología Social. Madrid: Prentice Hall. Décima edición.

Castillo V., E. (2009) Internet en Perú. Review 2009. [en línea] < http://www.slideshare. net/ ericholo61/internet-per $>$ [consulta: 12 julio 2010]

< http://www.internetworldstats.com/stats.htm [consulta: 10 noviembre 2011]

Fosk, A. (2011) Estado de Internet con un enfoque en el Perú. Interactive Advertising Bureau [en línea] < http://www.iabperu.com/descargas/Desc_2011920162111.pdf> [consulta: 10 noviembre 2011]

García L., C. (julio-diciembre, 2007) La percepción de utilidad del comercio electrónico. Enseñanza e investigación en Psicología 12 (002),pp 409-420.

Internet World Stats (2011) Internet usages statistics [en línea]

La Rosa Pinedo, Amaro (1983) Manual de Psicología Social. Lima: San Martín de Porres.

Lagarde M., H. (2001) México: el comercio electrónico. Un reto [en línea] Razón y Palabra. $\mathrm{N}^{\mathrm{0}} 20<\mathrm{http}$ ///www.razonypalabra.org.mx/anteriores/n20/20_hlagarde. html > [consulta: 12 julio 2010]

Lanzillota, A. (2007) Comprar a través de Internet, para algunos sí, pero para otros... [en línea] < http://www.mastermagazine.info/articulo/12071.php> [consulta: 12 julio 2010]

Schultz D.P. (1991) Psicología industrial. México: Mc Graw Hill.

Schiffman, L. \& Kanuk, L. (2000): Comportamiento del Consumidor. México: Prentice Hall. $7^{\mathrm{a}}$ ed.

Stanton, W.J., Michael, J.E \& Bruce, J.W. (2000) Fundamentos de Marketing. México, D.F.: Mc Graw-Hill/Interamericana Editores, S.A. de C.V.

Villar, A. M. (2004) Comercio electrónico: conceptos, recursos, estrategias. España: Ideas Propias Editorial. 


\section{ANEXOS}

\section{ESCALA DE ACTITUDES HACIA LA COMPRA A TRAVÉS DE INTERNET \\ FICHA TÉCNICA}

1. Nombre: Escala de actitudes hacia la compra a través de Internet.

2. Autor: Susan Pérez Alegría.

3. Administración: individual y colectiva.

4. Duración: variable entre 10 a 15 minutos

5. Aplicación: jóvenes y adultos

6. Material: cuadernillo de la prueba.

7. Tipificación: baremos en puntuaciones t.

\begin{tabular}{cc}
\hline CATEGORÍA & RANGO DE \\
PUNTUACIONES
\end{tabular}

\section{ESCALA DE ACTITUDES HACIA LA COMPRA A TRAVÉS DE INTERNET}

Susan Pérez A.

Lima 2010

Este es un cuestionario que le permitirá a usted efectuar algunas reflexiones respecto a las actitudes que tiene ahora, en relación a la compra a través de Internet.

No hay respuestas ni buenas ni malas, conteste según su propia opinión y no según crea que piensan los demás.

El cuestionario le llevará un tiempo no mayor a quince minutos. Le agradeceremos contestar a todas las preguntas dentro del tiempo señalado. 


\section{INSTRUCCIONES}

A continuación le presentamos una serie de enunciados para que usted coloque una " $\mathrm{x}$ " en el recuadro que mejor describa el grado de conformidad de su respuesta con cada uno de ellos.

\begin{tabular}{|c|c|c|c|c|c|c|}
\hline & 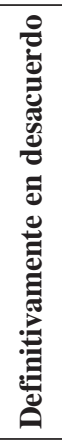 & 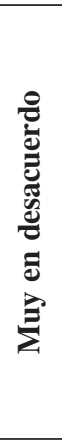 & 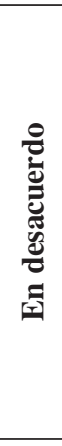 & 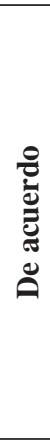 & 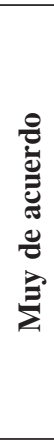 & 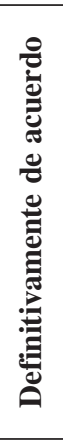 \\
\hline $\begin{array}{l}\text { 1. Al comprar a través de Internet tengo acceso a más } \\
\text { beneficios, como descuentos, promociones, sorteos, } \\
\text { etc. }\end{array}$ & & & & & & \\
\hline $\begin{array}{l}\text { 2. Es mejor comprar a través de Internet, porque así } \\
\text { no me expongo a los riesgos que implica manejar } \\
\text { efectivo. }\end{array}$ & & & & & & \\
\hline $\begin{array}{l}\text { 3. Tengo temor de realizar un pago virtual y luego no } \\
\text { recibir el producto o servicio ofrecido. }\end{array}$ & & & & & & \\
\hline $\begin{array}{l}\text { 4. Me desagrada que algunos productos se vendan } \\
\text { únicamente a través de Internet. }\end{array}$ & & & & & & \\
\hline $\begin{array}{l}\text { 5. Siento temor a hacer una transferencia económica } \\
\text { on-line para comprar algún artículo o pagar algún } \\
\text { servicio. }\end{array}$ & & & & & & \\
\hline $\begin{array}{l}\text { 6. Considero que los bancos brindan suficiente } \\
\text { seguridad en las transferencias económicas virtuales } \\
\text { para realizar compras on-line. }\end{array}$ & & & & & & \\
\hline $\begin{array}{l}\text { 7. Comprar a través de Internet me resulta más cómodo } \\
\text { que hacerlo yendo hasta una tienda, agencia u } \\
\text { oficina. }\end{array}$ & & & & & & \\
\hline $\begin{array}{l}\text { 8. Considero que comprando a través de Internet evito } \\
\text { las colas para realizar pagos, recoger productos, } \\
\text { comprobantes, etc. }\end{array}$ & & & & & & \\
\hline
\end{tabular}


9. Siento temor a ser estafado por una página web falsa o clonada al momento de comprar a través de Internet.

10. Considero que las compras de intangibles a través de Internet son seguras.

11. Una ventaja de comprar a través de Internet es que puedo hacerlo a cualquier hora y en cualquier lugar.

12. Considero que es peligroso usar una tarjeta bancaria para comprar a través de Internet.

13. Creo que las compras que se hacen a través de Internet son eficientes.

14. Considero que es riesgoso realizar una transferencia económica on-line para comprar algo a través de Internet.

15. El desarrollo de nuevas tecnologías de seguridad para transacciones económicas virtuales, me da la confianza de comprar en línea.

16. Considero que si compro a través de Internet ahorraré tiempo.

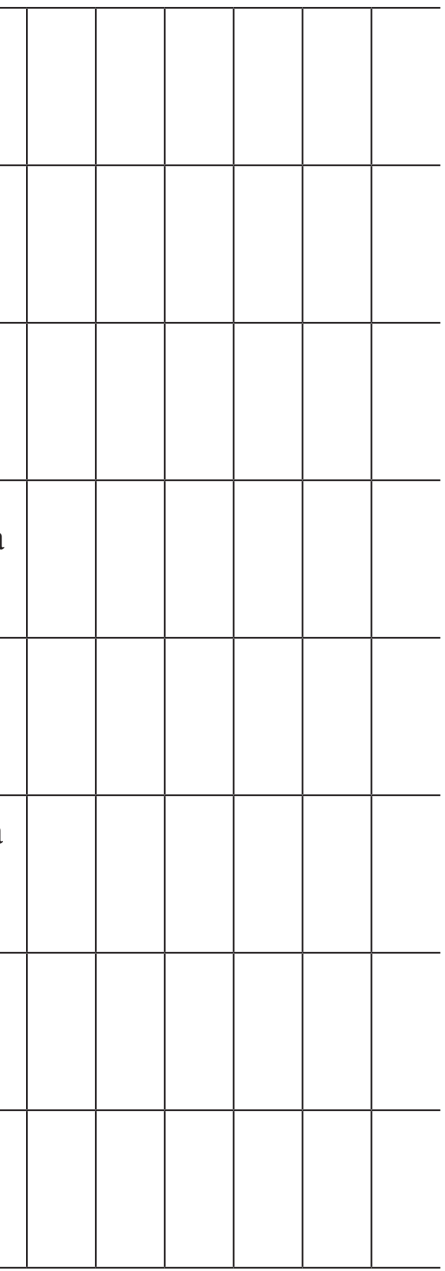

\section{Datos generales}

Edad:

$$
\text { Sexo: F( ) M ( ) }
$$

Especialidad:

Ciclo de estudios: 